\title{
A Novel Scheme with Adaptive Sampling for Better Spectrum Utilization in Cognitive Radios
}

\author{
Qun PAN, Xin ZHANG, Ruiming ZHENG, Yongyu CHANG, Dacheng YANG \\ Beijing University of Posts and Telecommunications, Beijing, China \\ Email: lionheartpq@gmail.com
}

\begin{abstract}
In cognitive radio (CR) networks, cognitive users need continuously monitor spectrum to decrease or avoid interference to primary users, yet attain a reasonable throughput. In this paper, we exploit a scheme to dynamically change the detection time duration to gain maximum throughput. Meanwhile the average detection time is kept as small as possible. The advantages of our approaches are approved by the deductions. Also the simulation results enable us to recognize the improved performance of our scheme over those ones with fixed detection time assignment.
\end{abstract}

Keywords: cognitive radio, cooperative sensing, average detection time, channel utilization ratio

\section{Introduction}

With the rapid development of wireless communication technology, the demand of spectrum resource is growing. The spectrum resource becomes rare due to the popularity of various new wireless applications. However, the recent survey shows a low spectral efficiency in the licensed band. The spectral holes in licensed band can be sensed and used to transmit information by employing cognitive radios technology [1]. The extra system throughput can be provided by cognitive radios without additional spectrum resource allocation. So the Federal Communications Commission (FCC) has recommended cognitive radio (CR) as the candidate for enhancing overall spectral efficiency. Cognitive radio [1] enables $\mathrm{CR}$ users (unlicensed users) to obtain the frequency bands with minimal interference to active primary users (licensed users) when they observe the bands allocated to the primary user are vacant and meanwhile detect the continuous spectrum sensing in CR networks. Therefore, as the heart of the cognitive radio techniques, the spectrum sensing is so critical that it determines the throughput and the agility of the CR networks.

One of the challenges of CR system's application is how to detect the primary user's signal quickly and correctly in order to avoid interference to the licensed network. The other is how to fully occupy the spectrum resources acquired by CR network. In this paper, a novel scheme is proposed to use the spectrum holes more efficiently. Meanwhile the interference to licensed network is controlled within an acceptable range. Our proposed scheme could increase spectrum efficiency in full extent in an applicable CR network. We consider the detection performance in the more practical scenario based on IEEE 802.22 WRAN system which uses cognitive radio technology as its key feature. The fast sensing and fine sensing process which utilize energy detection technology and feature detection technology respectively are both considered in our study. The cooperative sensing technology which can increase the sensing performance significantly is also involved in. The approaches of cooperative sensing technology [2-5] all use diversity technologies to improve effective SNR for essential.

The rest of this paper is written as follows. In Section 2 , we model the conventional detection scheme in mathematic way. In Section 3, we analyze the influence to channel utilization ratio made by time scheduling, and we propose a novel scheme of maximizing the channel utilization ratio. At last, we will conclude our study in Section 4

\section{System Model}

A practical sensing scheme is shown in Figure 1 [6]. There are two types of detection in the figure: the fast sensing (energy detection) and the fine sensing (feature detection). During these two types of detection time, all the CR users should cease their transmission in order to achieve high definition detection. Therefore, these detection periods are also called quiet periods.

The fast sensing processes are triggered periodically, and all the detection information from all CR users is summarized to give a final judgment. If the detection indicates that no primary user exists, the CR users will wait for next fast sensing process. On the other hand, the fine sensing process will be triggered. Usually, the time duration of fine sensing is much longer than that of fast sensing. Also the veracity of fine sensing is much better. Without loss of generality we assume the veracity of fine sensing is $100 \%$ all through the rest of the paper. 


\subsection{Modeling Individual Detection}

The binary hypothesis model is adopted in the study of spectrum sensing.

$$
\begin{aligned}
& \mathcal{H}_{0}: y(t)=n(t) \\
& \mathcal{H}_{1}: y(t)=h \cdot s(t)+n(t)
\end{aligned}
$$

where $y(t)$ is the receive signal by $\mathrm{CR}$ user at time $\mathrm{t}$. $n(t)$ is the Additive White Gaussian Noise (AWGN), and we assume $n(t)$ follows a standard normal distribution, which means the expectation is 0 and the variance is 1. $s(t)$ is the primary user's transmitting signal. $\mathrm{H}_{0}$ denotes the null hypothesis, which means there is no primary user signal in a certain spectrum band. $\mathrm{H}_{1}$ is the alternative hypothesis, which indicates that there exists primary user signal in that band. $h$ is the channel coefficient.

First, we consider local spectrum sensing (energy detection) at an individual CR user. We assume each energy detection interval contains $M$ samples. $\mathrm{j}$ denotes the sampling index. Then the statistic characteristic of the $i$-th CR user is given by:

$$
u_{i}=\left\{\begin{array}{cc}
\sum_{j=1}^{M} n_{i j}^{2} & \mathcal{H}_{b}, \\
\sum_{j=1}^{M}\left(h_{i} s_{i j}+n_{i j}\right)^{2} & \mathcal{H}_{1} .
\end{array}\right.
$$

We can see $u_{i}$ is the sum of $M$ independent Gau- ssian random variables' square, whose deviation is unit. So $u_{i}$ follows a central chi-square distribution with $M$ degree freedom when $\mathrm{H}_{0}$ becomes true, or else $u_{i}$ follows a non-central chi-square distribution.

$$
u_{i} \sim\left\{\begin{array}{cc}
\chi_{M}^{2} & \mathcal{H}_{0}, \\
\chi_{M}^{2}\left(\eta_{i}\right) & \mathcal{H}_{1} .
\end{array}\right.
$$

We defined the $\Gamma_{i}$ as the instantaneous SNR of the i-th CR user.

$$
\Gamma_{i}=\frac{1}{M} \sum_{j=1}^{M}\left|h_{i} s_{i j}\right|^{2}
$$

The non-centrality parameter $\eta_{i}=M \Gamma_{i}$.

If $M$ is large enough, the $u_{i}$ will approximately follow Gaussian distribution according to the Central Limit Theorem as follows:

$$
U_{i}=\frac{u_{i}}{M} \sim\left\{\begin{array}{c}
N(1, \sqrt{2 / M}) \\
N\left(1+\Gamma_{i}, \sqrt{2\left(1+2 \Gamma_{i}\right) / M}\right)
\end{array}\right.
$$

\subsection{Modeling Cooperative Detection}

Individual detection is not sufficient in a practical scenario due to the lower receive SNR of CR users. Hence, cooperative technology is involved in spectrum sensing as in [2-5]. We adopt a linear combine scheme which sums the different CR users' samples with weights in
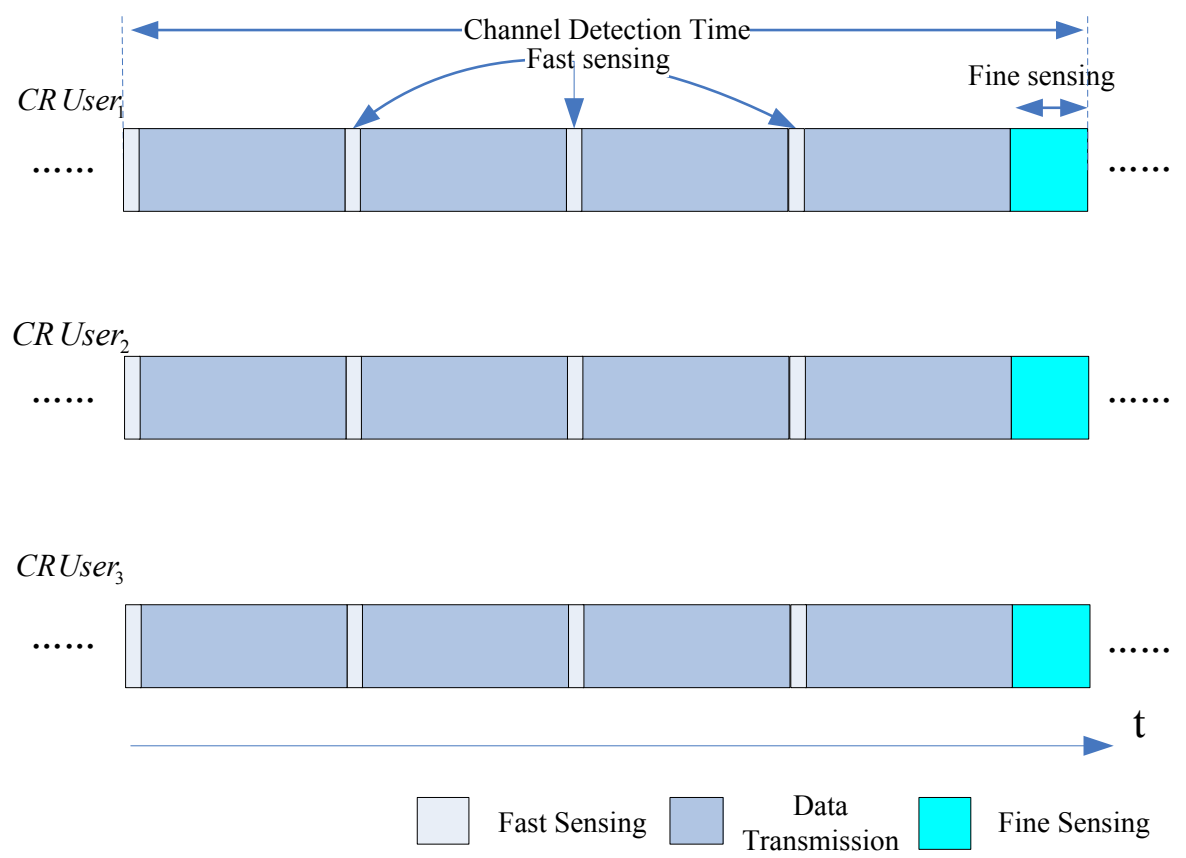

Figure 1. Detection process in cognitive radio network 
our cooperative sensing process. The weight coefficient corresponding to the $i$-th $\mathrm{CR}$ user is $w_{i}$, then the weighted summation is given by:

$$
\begin{gathered}
U=\sum_{i=1}^{N} w_{i} U_{i} \quad\left(\sum_{i=1}^{N} w_{i}^{2}=1,0<w_{i}<1\right) \\
N(1,2 / M)
\end{gathered}
$$

Let $\lambda$ be the decision threshold of energy detection. Therefore the probability of false alarm $P_{F}$, and that of detection $P_{D}$ are:

$$
\begin{gathered}
P_{F}=\mathrm{Q}\left(\frac{\lambda-\sum_{i=1}^{N} w_{i}}{\sqrt{2 / M \sum_{i=1}^{N} w_{i}^{2}}}\right) \\
P_{D}=\mathrm{Q}\left(\frac{\mathrm{Q}^{-1}\left(P_{F}\right)-\sqrt{M / 2} \sum_{i=1}^{N} w_{i} \Gamma_{i}}{\sqrt{\sum_{i=1}^{N} w_{i}^{2}\left(1+2 \Gamma_{i}\right)}}\right)
\end{gathered}
$$

So the decision threshold and the optimal weight coefficient can be given by (10) and (11) respectively [7]:

$$
\begin{gathered}
\lambda=\mathrm{Q}^{-1}\left(P_{F}\right) \sqrt{2 / M \sum_{i=1}^{N} w_{i}^{2}}+\sum_{i=1}^{N} w_{i} \\
w_{i}^{*}=\frac{\Gamma_{i}}{\sqrt{\sum_{i=1}^{N} \Gamma_{i}^{2}}}
\end{gathered}
$$

\section{System Performance Analysis and Proposed Scheme}

\subsection{Detection Performance}

The performance of the cooperative sensing is evaluated by receiver operating characteristics (ROC) which is composed of probability of false alarm and that of detection in plenty of bibliography [7][8]. But these two parameters can only indicate the system performance under some special conditions. Our analysis is shown as follows.

Excellent CR system should exit when the primary user is working on the target channel band, besides sufficiently use the spectrum when primary user is idle. Therefore the two objectives in CR system design are interference avoidance to primary user and full utilization of spectrum holes respectively. In order to decrease or avoid interference to the primary user, the detection sensitivity must be high enough for CR system, i.e. the existence of primary user's signal must be discovered as quickly as possible. If the detection process finds holes in spectrum, the time duration for detection must be as short as possible to leave more resource for data transmission.
We define channel utilization ratio (CUR) as the proportion of time used for data transmission accounting for the whole time when the primary user is idle [9]. $\xi_{E}$, $\xi_{D}$ and $\xi_{F}$ indicate the probabilities of energy detection, transmission and feature detection. The Markov state transition diagram is shown in Figure 2. So the corresponding Equation (12) is demonstrated as below.

$$
\left\{\begin{array} { c } 
{ \xi _ { T } ( 1 - P _ { F } ) + \xi _ { F } = \xi _ { E } } \\
{ \xi _ { T } P _ { F } = \xi _ { F } } \\
{ \xi _ { E } + \xi _ { T } + \xi _ { F } = 1 }
\end{array} \Leftrightarrow \left\{\begin{array}{c}
\xi_{E}=1 /\left(2+P_{F}\right) \\
\xi_{T}=1 /\left(2+P_{F}\right) \\
\xi_{F}=P_{F} /\left(2+P_{F}\right)
\end{array}\right.\right.
$$

So the CUR can be expressed by (13)

$$
\eta=\frac{T_{T} \xi_{T}}{\xi_{E} T_{E}+\xi_{T} T_{T}+\xi_{F} T_{F}}
$$

where $T_{E}, T_{T}$ and $T_{F}$ are the time duration of the three states. Let $T=T_{E}+T_{T}$. We define the sampling proportion (SP) as $\alpha=T_{E} / T$. So (13) can be transformed to (14).

$$
\eta=(1-\alpha) T / T+P_{F} T_{F}
$$

From (14) we can see it is the CUR that not only depends on $P_{F}$, but also $T$ and $\alpha$.

$$
\begin{aligned}
& P_{F}=\frac{(1-\alpha-\eta) T}{\eta T_{F}}>0 \\
& \eta<1-\alpha
\end{aligned}
$$

The average detection time (ADT) [10][11] indicates the average time interval from the primary user's presence until it is found by CR users. We assume the probability of detection in each sensing period is equal to $P_{D}$, when the primary user is present. The final $\mathrm{H}_{1}$ decision must be after feature detection, so the ADT required by CR user to detect the primary user can be given by (16):

$$
T_{D}=T \sum_{k=1}^{\infty} k P_{D}\left(1-P_{D}\right)^{k-1}+T_{F}=\frac{T}{P_{D}}+T_{F}
$$

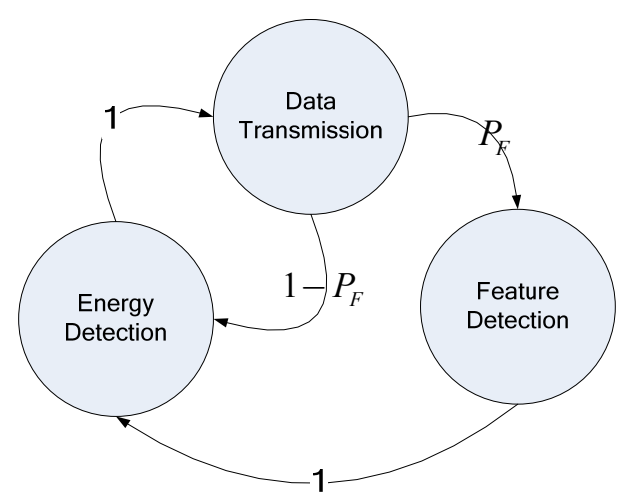

Figure 2. State transition diagram of CR system when no primary user is active 


\subsection{Maximizing the Channel Utilization Ratio}

The interference provided by CR network should be firstly small enough, so that primary user can work normally. In order to limit the interference to primary user, the ADT should have a maximum target. So we will discuss the scheme which can maximize the CUR, and at the same time the target of ADT can be realized.

The sampling number $M=W T_{E}=W T \alpha$ according to the Nyquist sampling Theorem, where $W$ is the sensing bandwidth. (17) is acquired in terms of (9) and (16):

$$
P_{F}=\mathrm{Q}\left(\sqrt{\sum_{i=1}^{N} w_{i}^{2}\left(1+2 \Gamma_{i}\right)} \mathrm{Q}^{-1}\left(\frac{T}{T_{D}-T_{F}}\right)+\sqrt{W T \alpha} \sum_{i=1}^{N} w_{i} \Gamma_{i}\right)
$$

The CUR can be given by (18) which is combined by (14) and (17).

$$
\eta=\frac{(1-\alpha) T}{\mathrm{Q}\left(\sqrt{\sum_{i=1}^{N} w_{i}^{2}\left(1+2 \Gamma_{i}\right)} \mathrm{Q}^{-1}\left(\frac{T}{T_{D}-T_{F}}\right)+\sqrt{W T \alpha} \sum_{i=1}^{N} w_{i} \Gamma_{i}\right) T_{F}+T}
$$

(18) shows that there are too many parameters which could influence the CUR. The bandwidth $W$ is fixed and it depends on the spectrum specification of primary network. The SNR $\Gamma_{i}$ of each CR user depends on the radio scenario and the receive equipment of $C R$ user. The time duration of feature detection $T_{F}$ depends on the character of primary user's signal. Conventionally the frequency of the fast detection $1 / T$ is predefined. Therefore, the only parameter which can be adjusted is the $\alpha$, i.e. the time duration of each fast sensing can be changed to optimize the CUR.

Intuitively if the sensing duration is too short, and simultaneously the ADT can be guaranteed, the probability of false alarm will be raised. As a result, the unnecessary feature detection will be frequently triggered. The unnecessary feature detection will waste a large amount of time which can be used for information transmission. But if the sensing duration is too long, the spectrum resources would be wasted by fast sensing process. Hence, there must be an optimal sensing duration which can maximize the CUR. From all above, it is obvious that the optimal scheme is to integrate information from all CR users, and then calculate the optimal sensing duration to inform each CR user.

To prove the existence of optimal sensing duration, the numerical simulation is brought about in our study. The simulation parameters are shown in Table 1. [6].

In Figure 3 the maximum CUR can be achieved when the sampling number $M$ is at an appropriate value. However, the optimal sampling number is not fixed among

Table 1. The simulation parameters

\begin{tabular}{cc}
\hline Parameters & Value \\
\hline Average Detection Time & $0.5 \mathrm{~s}$ \\
Sensing Interval & $10 \mathrm{~ms}$ \\
Feature Detection Time & $100 \mathrm{~ms}$ \\
Instantaneous SNR & $-24 \mathrm{~dB},-20 \mathrm{~dB},-16 \mathrm{~dB},-12 \mathrm{~dB},-8 \mathrm{~dB}$ \\
Sensing Bandwidth & $6 \mathrm{MHz}$ \\
CR User Number & 5 \\
\hline
\end{tabular}

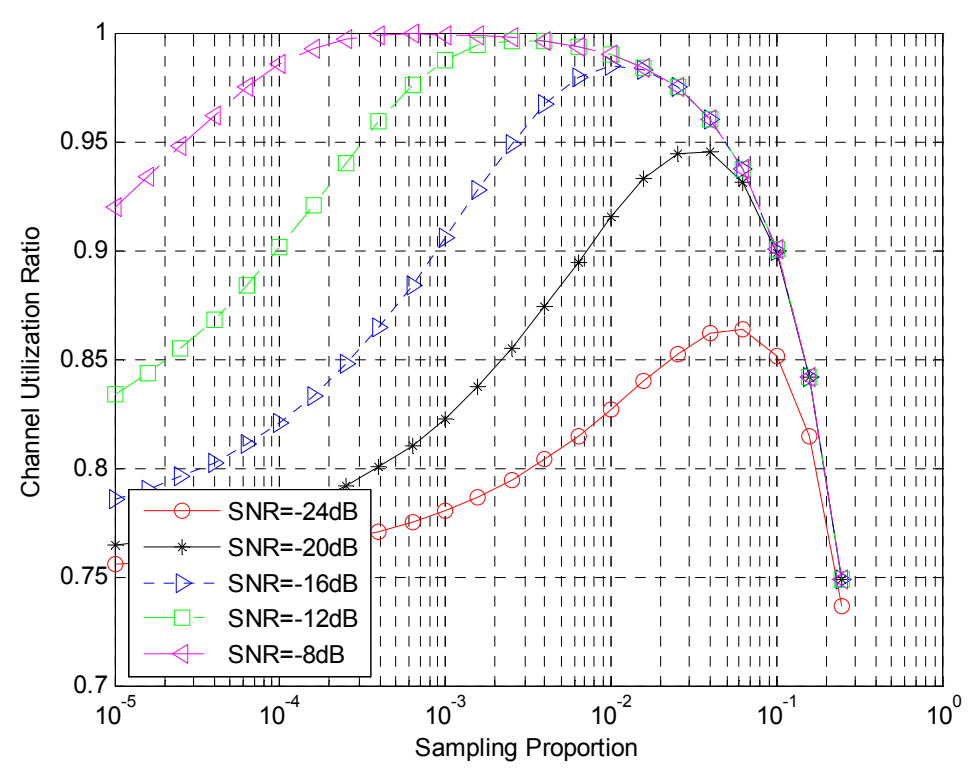

Figure 3. The channel utilization ratio vs. sampling proportion 


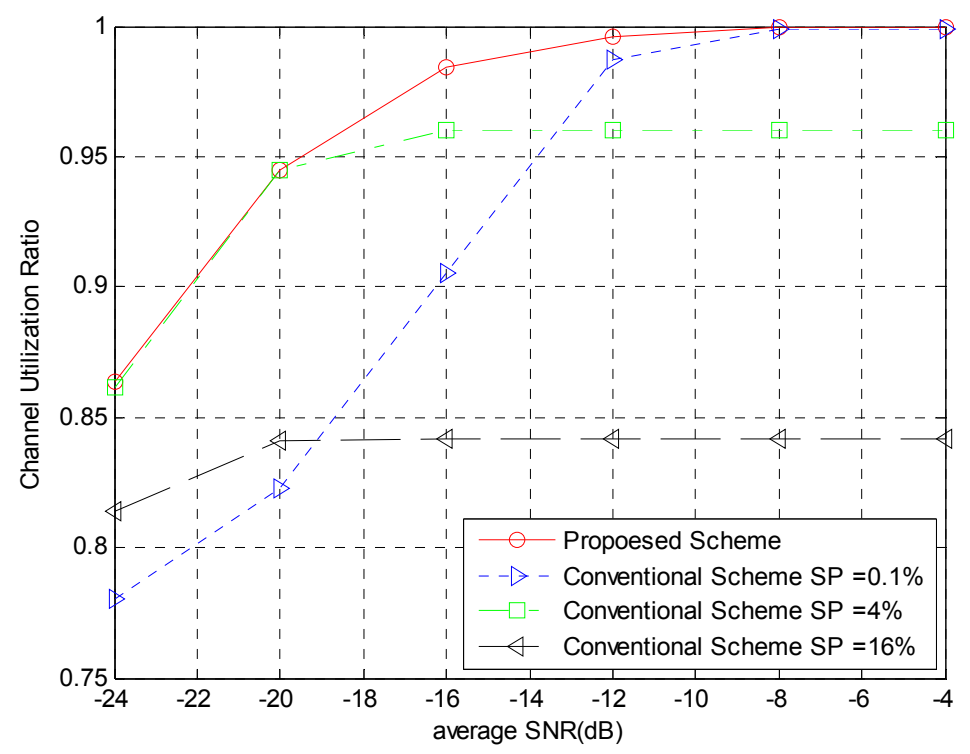

Figure 4. The channel utilization ratio vs. average SNR

different SNR scenarios. Although the optimal sampling number $M$ cannot be calculated easily, the One-dimensional Search Method can deal with the problem. It is clear that in order to utilize the spectrum resource more efficiently, the sampling number must be dynamically adjusted according to the SNRs of CR users. Therefore, we propose a novel sensing time schedule for CR system, which is called dynamic adaptive sensing duration scheme:

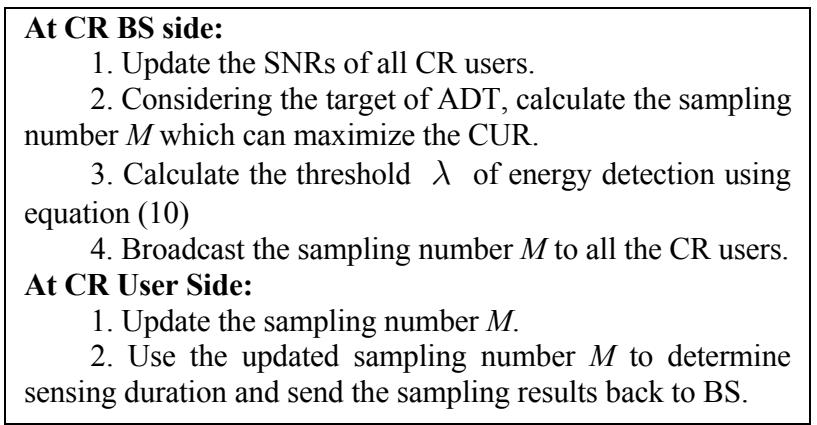

In Figure 4 we compare our proposed scheme with three conventional ones whose sampling numbers are different from each other but not dynamically changed. It is obvious that the conventional scheme can gain a high CUR only when the SNRs are at an appropriate level. However the proposed scheme can always maximize the CUR, in other words the proposed scheme reaches the upper bound of the CUR for detection in CR system.

\section{Conclusions}

We have focused on how to maximize the CUR and produce interference small enough to primary network.
We deduce the relationship between conventional ROC which is composed by Prob. of detection and that of false alarm and our proposed ROC which contains CUR and ADT. Our proposed scheme shows that the CUR can be maximized by optimizing time duration of energy detections according to different SNR conditions.

\section{REFERENCES}

[1] MITOLA J and MAGUIRE G Q. Cognitive radio: Making software radios more personal. Personal Communications, IEEE, 1999, 6: 13-18.

[2] GANESAN G and YE L. Cooperative spectrum sensing in cognitive radio, Part I: Two user networks. IEEE Transactions on Wireless Communications, 2007, 6: 2204-2213.

[3] UNNIKRISHNAN $J$ and VEERAVALLI V V. Cooperative spectrum sensing and detection for cognitive radio. In IEEE Global Telecommunications Conference, GLOBECOM'07, 2007, 2972-2976.

[4] ZHI Q, SHUGUANG C, and SAYED A H. Optimal linear cooperation for spectrum sensing in cognitive radio networks. IEEE Journal of Selected Topics in Signal Processing, 2008, 2: $28-40$.

[5] WeI Z, MALLIK R K, and LETAIEF K. B. Cooperative spectrum sensing optimization in cognitive radio networks. In IEEE International Conference on Communications, ICC'08, 2008, 3411-3415.

[6] IEEE 802.22-06/0003r3. A PHY/MAC proposal for IEEE 802.22WRAN systems, Part 2: The cognitive MAC. Mar. 2006.

[7] MA J and LI Y G. Soft combination and detection for cooperative spectrum sensing in cognitive radio networks. In Global Telecommunications Conference, GLOBECOM'07, IEEE, 2007, 3139-3143.

[8] TAHERPOUR A, KENARI N M, and JAMSHIDI A. Efficient cooperative spectrum sensing in cognitive radio networks. In 
IEEE 18th International Symposium on Personal, Indoor and Mobile Radio Communications, PIMRC'07, 2007, 1-6.

[9] SOOK J W, J. GEUN D, H J AE, K. GWANGZEEN, and SUN S M. An efficient quiet period management scheme for cognitive radio systems. IEEE Transactions on Wireless Communications, 2008, 7: 505-509.
[10] GANESAN G, LI Y, and LI S. Spatiotemporal sensing incognitive radio networks. In IEEE 18th International Symposium on Personal, Indoor and Mobile Radio Communications, PIMRC'07, 2007, 1-5.

[11] GANESAN G, YE L, BING B, and LI S Q. Spatiotemporal sensing in cognitive radio networks. IEEE Journal on Selected Areas in Communications, 2008, 26: 5-12. 